\title{
An Agent-Based Simulation Model for Urban Traffic System
}

\author{
Ali Bazghandi \\ School of Computer and IT Engineering, Shahrood University of Technology \\ PO box 316, Shahrood, Iran \\ Tel/Fax: 98-27-3339-3580 E-mail: bazghandi@shahroodut.ac.ir \\ Ali A. Pouyan, member IEEE \\ School of Computer and IT Engineering, Shahrood University of Technology \\ PO box 316, Shahrood, Iran \\ Tel/Fax: 98-27-3339-3580 E-mail: apouyan@ieee.org
}

Received: May 21, 2011

Accepted: June 23, 2011

doi:10.5539/cis.v4n4p72

\begin{abstract}
In this paper, we propose a model for vehicle traffic based on multi-agent systems and account suppositions and its issues. Traffic is an ever-growing problem as population and the number of drivers around the world increase exponentially. Previously, fluid flow models have been used in an attempt to model traffic. Based on recent studies, only agent based models can accurately model a traffic scenario. This is because small perturbations could have a butterfly-like effect, which causes a rapid change in the entire system.
\end{abstract}

Keywords: Traffic, Agent based modeling, Simulation, Deadlock, Crash, Performance

\section{Introduction}

\subsection{Agent Based Modeling}

A model is a simplified representation of reality. In other words, a model consists of one or more processes that are believed to occur in the real-world (Longley, P.A. and Batty, M. 2003). Based on the nature of investigation, several types of models could exist for a single real world scenario. A model can be constructed as a computer program that uses (usually to some degree) a simplified digital representation of one or more aspects of the real-world, transforming them to create a new representation.

There is no universal agreement on the precise definition of the concept of "agent". Definitions of agent tend to agree on more points than the differences on constituent parts (Bonabeau, E. 2002).

The concept of an agent as a software entity, is meant to be a tool for analyzing a system, not an absolute classification where entities can be defined as agents or non-agents (Russell, S. and Norvig, P. 2003). For example, some modelers consider any type of independent component (i.e. software, model, individual, etc.), to be an agent. For a detailed version of agent theory the reader is referred to (Russell, S. and Norvig, P. 2003).

Agent based modeling ( $\mathrm{ABM}$ ) is not the same as object-oriented simulation, although the object-oriented paradigm provides a suitable medium for the development of agent-based models. For this reason, ABM systems are invariably object-oriented(Christian, J., E, Castle. 2006). Agent-based models are comprised of multiple, interacting agents situated within a model or simulation environment. A relationship between agents is specified, linking agents to other agents and/or other entities within a system. Relationships may be specified in a variety of ways, from simply reactive (i.e. agents only perform actions when triggered to do so by some external stimulus e.g. actions of another agent), to goal-directed (i.e. seeking a particular goal). The behavior of agents can be scheduled to take place synchronously (i.e. every agent performs actions at each discrete time step), or asynchronously (i.e. agent actions are scheduled by the actions of other agents, and/or with reference to a clock).

In a modeling context, agent-based models can be used as experimental media for running and observing agent-based simulations. The ability to simulate individual actions of many diverse agents and measure the resulting system behavior and outcomes over time (e.g. changes in patterns of pedestrian emergency egress), means agent-based models can be useful tools for studying the effects on processes that operate at multiple 
scales and organizational levels, and their effects (Brown, D.G. 2006). In particular, the roots of ABM are within the simulation of human social behavior and individual decision-making domain (Bonabeau, E. 2002).

\subsection{Traffic simulation}

As stated earlier, a multi-agent system (MAS) is a system which consists of a number of agents interacting with each other. Though the local interaction among agents is simple, it emerges complex phenomena as a whole. Such a system is called complex systems and a traffic flow is an example of the complex systems, which is emerged from vehicle agents (Kosuke Ono, Takashi Hata, Toyofumi Maetani, Masateru Harao and Kouichi Hirata, T. Washio et al. 2006). Complex system by definition has non-linearity. Non-linearity exists in MAS and traffic flow mechanism. The agents used in the microscopic level contain the following types: vehicle agent, road agent, intersection agent and signal agent. In the simulation model vehicle agents are mobile agents.

Traffic jams are caused when drivers don't have access to information about road systems ahead of them. If the vehicles and the actual road system itself have information, it can respond and react according to that information and impose certain limitations on the vehicles to alleviate traffic. Currently, there is research on optimization of traffic and variable speed limit areas for places with high frequency traffic jams. Using a model, the extent that variable speed limit and other changing traffic laws can be measured to see if implementing that technology is a viable solution to traffic jams (Timmy Galvin. 2005).

Here, we propose a model that responds on vehicle traffic and investigate issues and results due to changing some traffic parameters. We need to have an accurate model of driver behavior and the ability of collecting data and analyzing the situation. The model can be applied generically. Vehicles on the road will have their own properties such as location, speed, acceleration. Some of these properties can be defined through the simulation stages. In this model vehicle collisions have not been considered.

\section{Model Description}

\subsection{Principals}

Our simulation that will model different traffic scenarios will depict traffic jams and use various methods to respond according. This will be viewed using an overhead view of the system. We alter some parameters such as: speed limit, acceleration, traffic jams and traffic lights to retard deadlock.

We hope to show some results graphically on the change in deadlock and flow versus density and other parameters.

In this model we consider a city with traffic parameters. Streets have various lanes. We design streets layout. A lot of vehicles cross along streets. Various kinds of vehicles have different size, acceleration, speed.

Naturally traffic jam is more in center of city. To import this factor, we define center-jam-coef factor which differ from 1 to 2. This factor is used in decision making by agents in intersections for choosing a lane. The decision factor is a normally distributed random floating point number with the mean zero and standard-deviation $\sqrt{x^{2}+y^{2}}$ :

decision $=$ abs $\left(\right.$ random - normal $\left.\left(0 \sqrt{x^{2}+y^{2}}\right)\right)$ Then decision factor is compared according to below expression:

$$
\text { decision } \stackrel{?}{\leq}\left(\frac{\sqrt{x^{2}+y^{2}}}{\text { center_jam_coef }}\right)
$$

To set of vehicle agents speed, we consider some parameters:

- Being or not an agent on the cell in front of the agent. if there are vehicle agents in front of the turtle, slow down otherwise, speed up (not exceeding the speed limit)

- Being or not red light in the intersections.

- Acceleration

Traffic light in the intersections helps to control Traffic jams and avoid accident. Figure 2.1 shows one intersection and Figure 2.2 shows our simulation environment.

Deadlock as usual occurrence in traffic, changes macroscopic variables such as: flow, speed and density. If average density, flow and speed are expressed with $\mathrm{k}, \mathrm{q}$ and $\mathrm{v}$ then: 


$$
q=f(k)=v \cdot k
$$

Figure 2.3, shows relations between $\mathrm{k}$, $\mathrm{q}$ and $\mathrm{v}(\mathrm{Xiao}-$ liang Ma. 2007).

\subsection{Deadlock}

Several deadlock situations may occur in real world traffic. Two deadlock scenarios are shown in Figure 2.4.

In these situations deadlock can be solved (avoided in real world) by certain strategies, for example by killing a "vehicle agent". In our model the deadlock shown in Figure 2.4 can be resolved.

Deadlock situations can cause a decrease in local traffic flow speed. By changing some control parameters (in the simulation) deadlock behavior can be investigated. Here, we consider traffic green (or red) time as control variables. Changes in control variables affect traffic flow via macroscopic variables.

\subsection{Accident and crash}

A number of studies have investigated the relationship between speed and crash risk(Kloeden CN, McLean AJ, Moore VM, Ponte G. 1997). It is well-established that once a crash has occurred, the severity of the injuries sustained by the individuals involved is an increasing function of vehicle speed. The relationship is non-linear, an increase in vehicle speed produces a proportionately greater increase in injury severity. For example, Joksch showed that compared to the risk of an occupant fatality following involvement in a crash at $40 \mathrm{mph}$, the risk of a fatality was 2.5 times greater at $60 \mathrm{mph}, 6$ times greater at $70 \mathrm{mph}$, and approximately 20 times greater at 80 mph (Joksch HC. 1975). Injury severity is, in fact, more directly related to the change in velocity experienced during the crash, but change in velocity tends to increase with increasing pre-crash speed (O'Day J, Flora J. 1982).

According to the results of Solomon, the involvement rates as a function of deviation from mean speed, to overcome irregularities due to the highway sections having a range of speed limits and mean speeds. Under this configuration the involvement rates were again U-shaped, being maximum for vehicles with speeds of more than $35 \mathrm{mph}$ below the average, minimum for speeds of 5 to $10 \mathrm{mph}$ above the average, and somewhat elevated for further deviations above the average. These results are depicted in Figure 2.5 (Kloeden CN, McLean AJ, Moore VM, Ponte G. 1997)(Solomon D. 1964).

\section{Case study}

In our case study we consider a network of streets with 25 intersections. Every street has two lanes for two opposite directions. All intersections have traffic lights. Green (Red) time for all traffic lights is the same but all of them not alter simultaneously. $190 \pm 10$ vehicle agents move in the streets. Moving area for agents are 1520 vehicles.

At the first, crashes are ignored in our system. We define performance as a function of speed mean.

With altering red (green) time of lights we get these results (Table 3.1):

In the above tables $\mathrm{T}$ is red (green) time, $\mathrm{dt}$ is time between two deadlocks and $\mathrm{v}$ is speed mean for every scenarios. ( $\mathrm{T}=0$ means light power is off).

We define performance function like this: $P \cong V_{\text {mean }} * C^{-1}$

Where $\mathrm{C}$ is the crash coefficient.

Figure 3.1 shows Relation between red (green) time and performance.

Also in figure 3.2 you can see relation between red (green) time and mean of speed with 200 vehicles.

We can anticipate that above curve is attenuated. Maximum and minimum values repeated alternatively (Figure 3.3).

From tables 3.1, 3.2, 3.3, 3.4 and figures $3.1(\mathrm{a}, \mathrm{b}, \mathrm{c}, \mathrm{d})$ we find that when vehicles are multiplied by n, performance decrease proportionality. On the other hand with red time (T) constant we perceive:

$$
P_{n}=f\left(k, p_{0}\right) \cong P_{0} \frac{1}{2^{\frac{k_{n}}{k_{0}}}}
$$

Where $\mathrm{k}_{0}$, kn are average densities in two cases (initial state and state $n$ ). $P_{0}$ and $P_{n}$ represent performance in initial state and state $n$, respectively. This function shows that without changes in red or green time, an increase in density decreases performance, exponentially. 


\section{Conclusions and future works}

In this paper, we introduced a simulation model based on agent theory for urban traffic system. Fixed vehicle density in traffic causes the overall average of speed and performance decreases when red (green) time increases. We have shown that without changes in red or green time, increasing in car density causes a decrease in performance, exponentially. We have also shown that red (green) time does not influence on occurring the deadlock situation. Change in some parameters such as: center-jam-coef (a coefficient for traffic jam in a city downtown) and investigation of issues in this area can be considered as future trend in our research.

\section{References}

Bonabeau, E. (2002). Agent-Based Modelling: Methods and Techniques for Simulating Human Systems", Proceedings of the National Academy of Sciences of the United States of America (PNAS), 99(3): 7280-7287. 2002.

Brown, D.G. (2006). Agent-Based Models, in Geist, H. (ed.) The Earth's Changing Land: An Encyclopedia of Land-Use and Land-Cover Change, Greenwood Publishing Group, Westport, pp. 7-13. 2006.

Christian, J., E, Castle. (2006). Principles and Concepts of Agent-Based Modeling for Developing Geospatial Simulations. (Sep 06).

Joksch HC. (1975). An empirical relation between fatal accident involvement per accident involvement and speed. Accident Analysis and Prevention; 7(2): 129-132. 1975.

Kloeden CN, McLean AJ, Moore VM, Ponte G. (1997). Traveling Speed and the Risk of Crash Involvement. Volume 1 - Findings, November 1997.

Kosuke Ono, Takashi Hata, Toyofumi Maetani, Masateru Harao and Kouichi Hirata, T. Washio et al. (2006). Development of a Multi-Agent Based Generic Traffic Simulator. (Eds.): JSAI 2005 Workshops, LNAI 4012, pp. 249-260, 2006. c_Springer-Verlag Berlin Heidelberg 2006.

Longley, P.A. and Batty, M. (2003). Prologue: Advanced Spatial Analysis: Extending GIS, (eds.), Advanced Spatial Analysis: The CASA Book of GIS, 2003. ERSI Press, Redlands, California.

O'Day J, Flora J. (1982). Alternative measures of restraint system effectiveness: interaction with crash severity factors. Warrendale, Pennsylvania: Society of Automotive Engineers. Technical Paper No.: 820798. 1982.

Russell, S. and Norvig, P. (2003). Artificial Intelligence: A Modern Approach, Prentice Hall, USA. 2003.

Solomon D. (1964). Accidents on main rural highways related to speed, driver and vehicle. Washington, DC: US Department of Commerce \& Bureau of Public Roads. 1964. P 10.

Timmy Galvin. (2005). A Traffic Simulation Model Allowing For Wide-ranged Vehicle Communication. Proceedings of the Alife IV. 2005.

Xiao-liang Ma. (2007). Traffic stream, car-following and vehicle arrival models, Introduction to Traffic Flow Theory. 2007 Fall. 
Table 3.1 Relation between speed mean, red time and time between two deadlocks (density is 200 vehicles)

\begin{tabular}{|c|c|c|c|c|c|}
\hline $\mathbf{T}$ & 0 & 5 & 10 & 15 & 20 \\
\hline dt & 20.76 & 1708 & 1176 & 771.3 & 573.8 \\
\hline $\mathbf{V}$ & 0.653 & 0.489 & 0.44 & 0.424 & 0.405 \\
\hline $\mathbf{T}$ & 25 & 30 & 35 & 40 & 45 \\
\hline dt & 555.4 & 653.9 & 1022 & 907.2 & 950.5 \\
\hline $\mathbf{V}$ & 0.379 & 0.383 & 0.405 & 0.393 & 0.382 \\
\hline $\mathbf{T}$ & 50 & 55 & 60 & 65 & \\
\hline dt & 890.9 & 1057 & 1095 & 1056 & \\
\hline $\mathbf{V}$ & 0.366 & 0.352 & 0.351 & 0.332 & \\
\hline
\end{tabular}

Table 3.2 Relation between speed mean, red time and time between two deadlocks (density is 400 vehicles)

\begin{tabular}{|ccccccc|}
\hline $\mathbf{T}$ & $\mathbf{0}$ & $\mathbf{5}$ & $\mathbf{1 0}$ & $\mathbf{1 5}$ & $\mathbf{2 0}$ \\
$\mathbf{d t}$ & 0.3691 & 0.3047 & 0.2558 & 0.2368 & 0.191 \\
$\mathbf{v}$ & 7.84 & 408 & 248 & 150 & 130 \\
\hline $\mathbf{T}$ & 25 & 30 & 35 & 40 & 45 \\
$\mathbf{d t}$ & 0.16 & 0.17 & 0.15 & 0.13 & 0.127 \\
$\mathbf{v}$ & 145 & 193 & 145 & 174 & 167 \\
\hline
\end{tabular}


Table 3.3 Relation between speed mean, red time and time between two deadlocks (density is 600 vehicles)

\begin{tabular}{|cccccc|}
\hline $\mathbf{T}$ & $\mathbf{0}$ & 5 & 10 & 15 & 20 \\
$\mathbf{d t}$ & 0.27 & 0.218 & 0.18 & 0.15 & 0.136 \\
$\mathbf{v}$ & 4 & 235 & 146 & 110 & 88 \\
\hline $\mathbf{T}$ & 25 & 30 & 35 & 40 & 45 \\
$\mathbf{d t}$ & 0.131 & 0.1 & 0.085 & & \\
\hline $\mathbf{v}$ & 81 & 140 & 125 & & \\
\hline
\end{tabular}

Table 3.4 Relation between speed mean, red time and time between two deadlocks (density is 800 vehicles)

\begin{tabular}{|cccccc|}
\hline T & 0 & 5 & 10 & 15 & 20 \\
\hline dt & 0.17 & 0.161 & 0.135 & 0.113 & 0.105 \\
v & 4 & 125 & 105 & 68 & 63 \\
\hline T & 25 & 30 & 35 & 40 & 45 \\
\hline dt & 0.084 & 0.078 & 0.075 & 0.065 & \\
\hline & 67 & 69 & 66 & 68 & \\
\hline
\end{tabular}

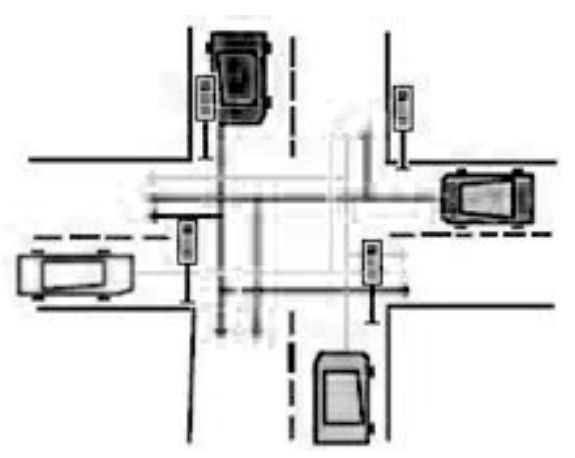

Figure 2.1 One intersection 


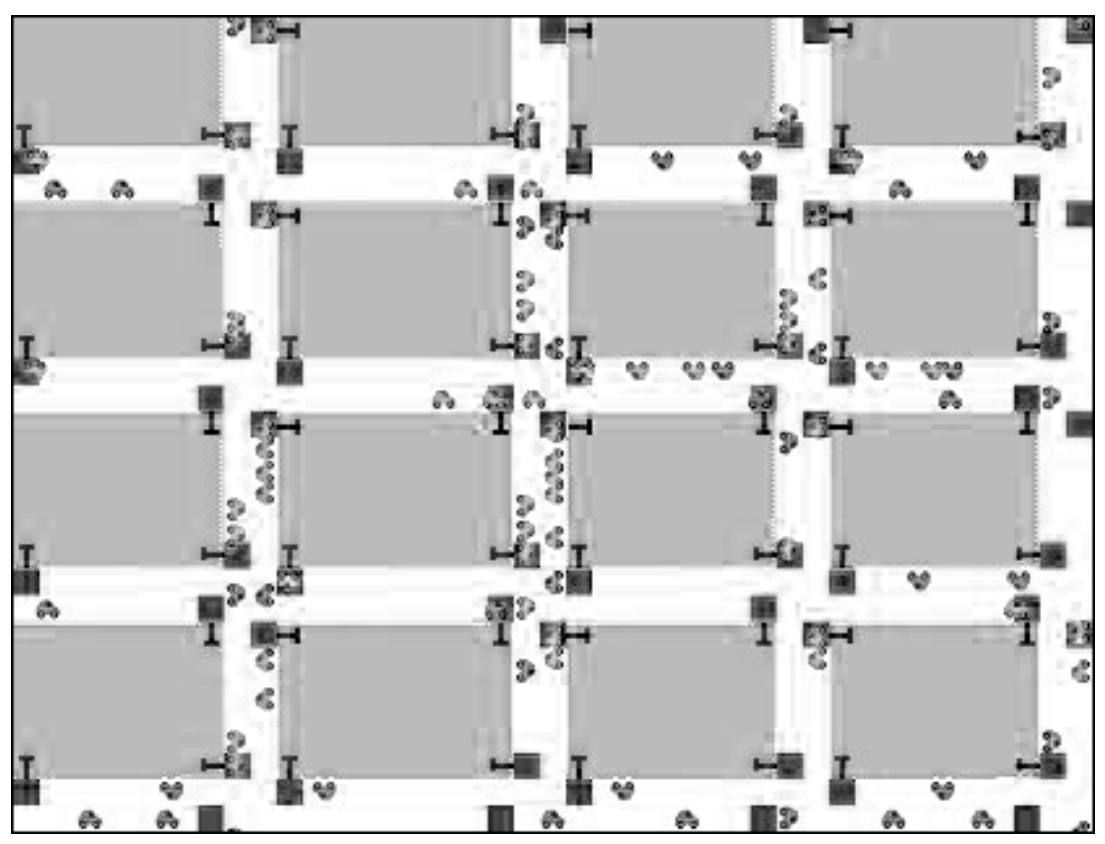

Figure 2.2 Our simulation environment 

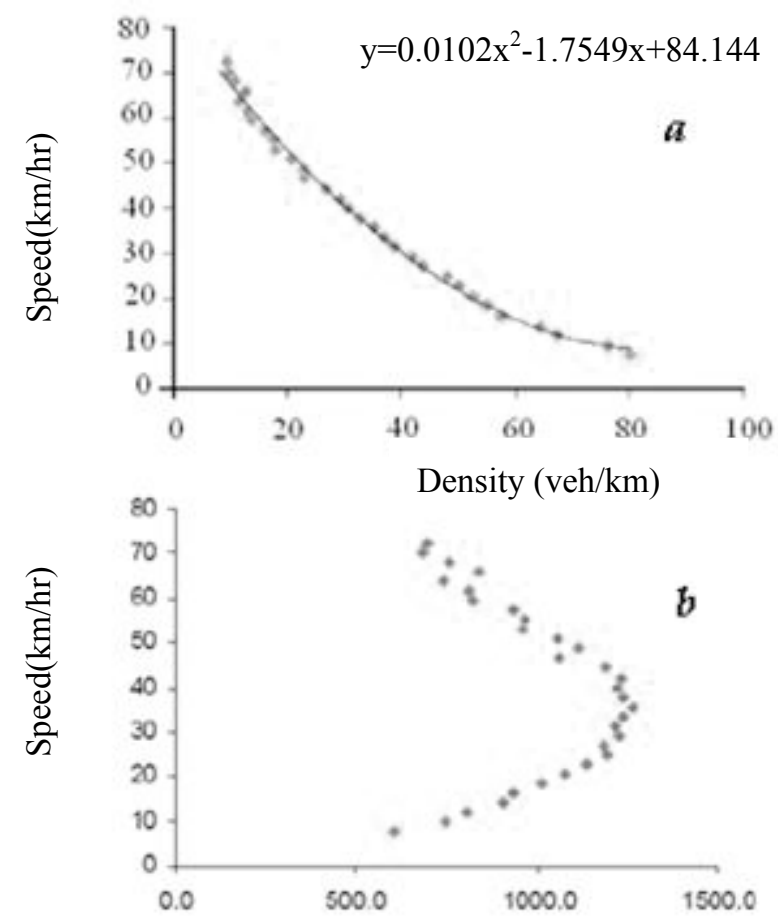

Flow(veh/hr)

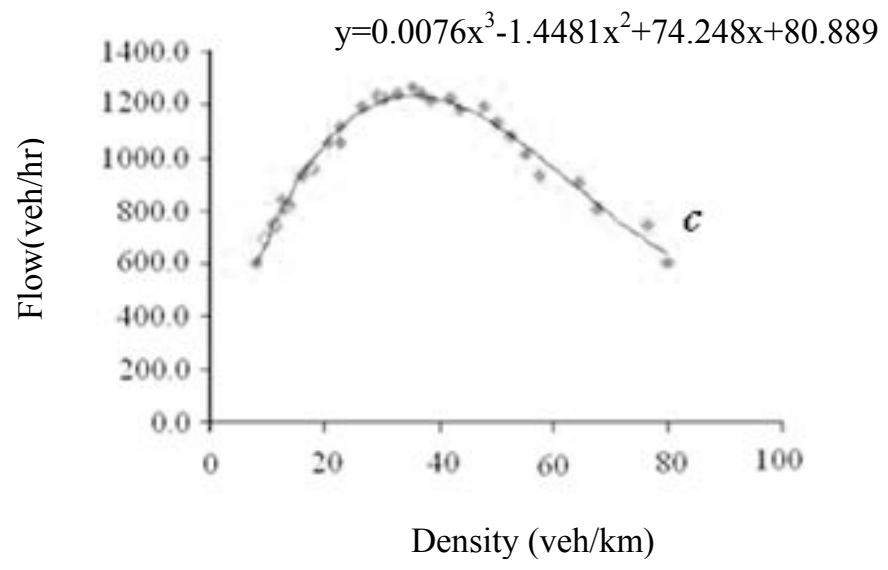

Figure 2.3 Relations between three macroscopic variables k, q and v 

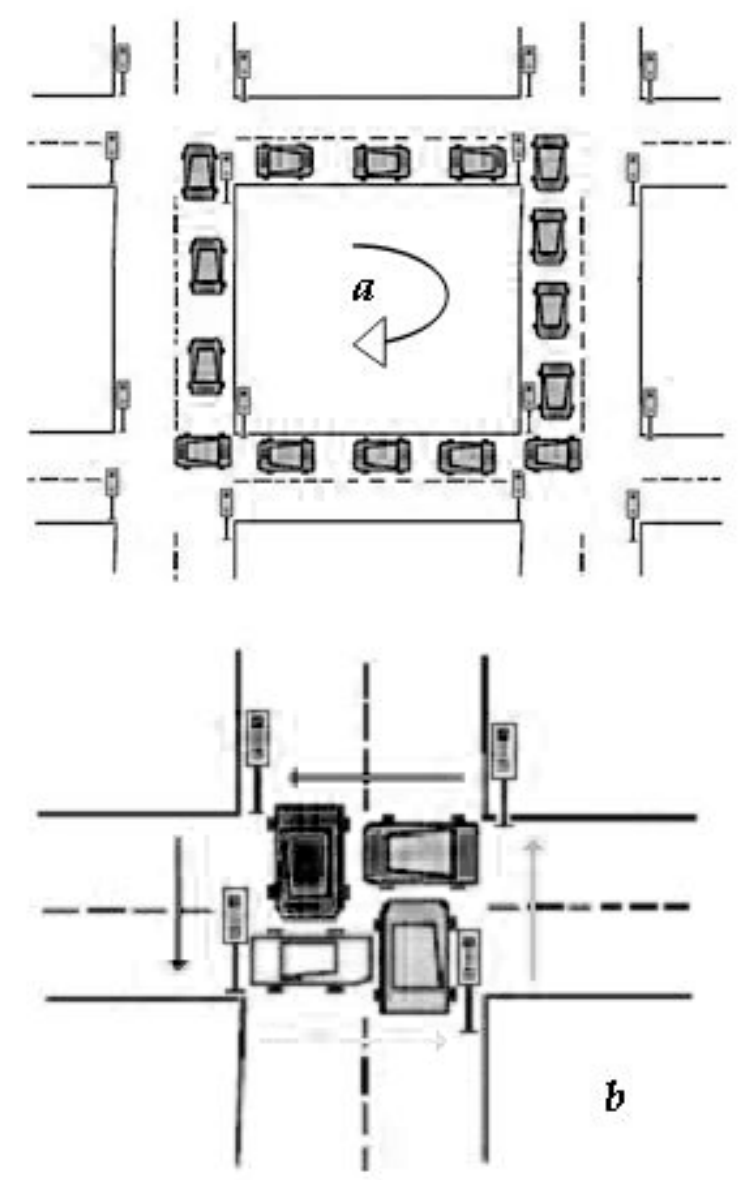

Figure 2.4 Some deadlock scenarios

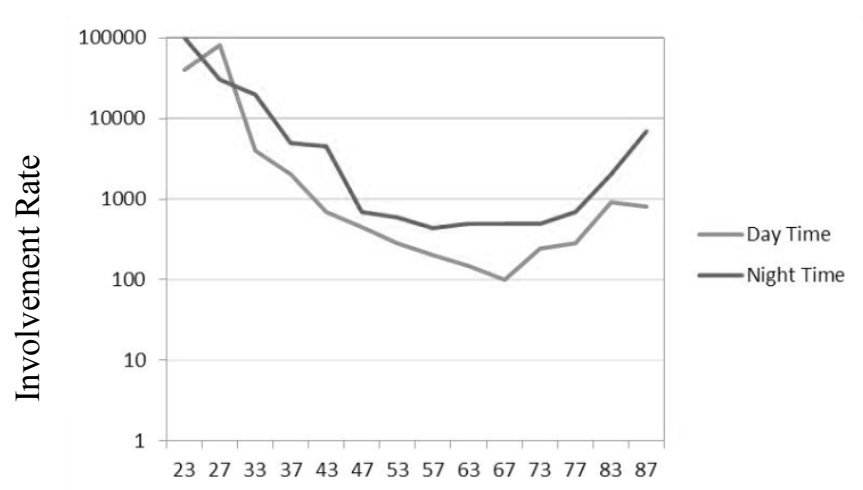

Figure 2.5 Accident involvement rate by travel, speed, day and night 
a)

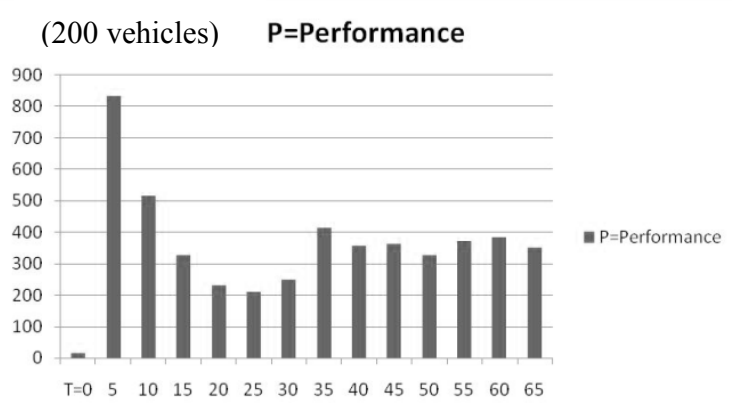

b)

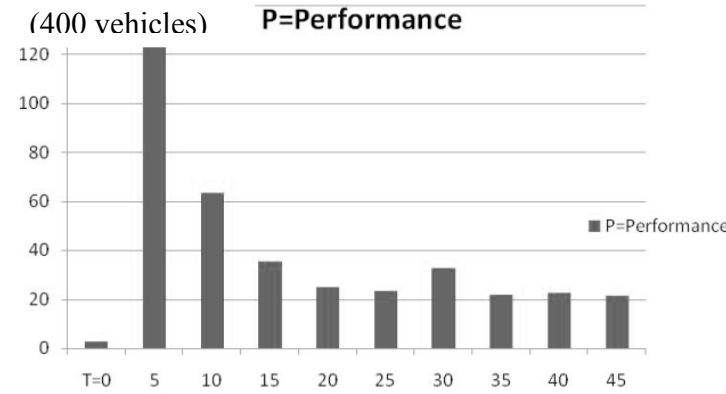

c)

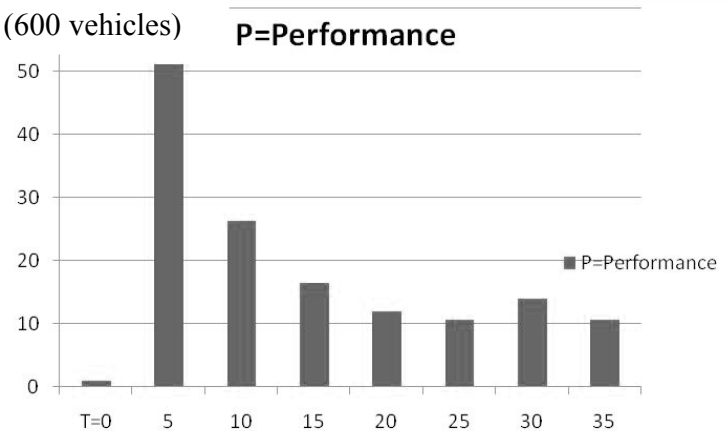

(800 vehicles) P=Performance

d)

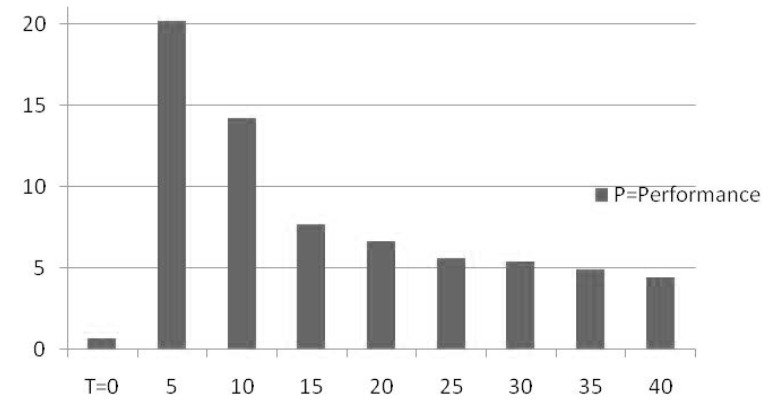

Figure 3.1 Relation between red (green) time and performance 


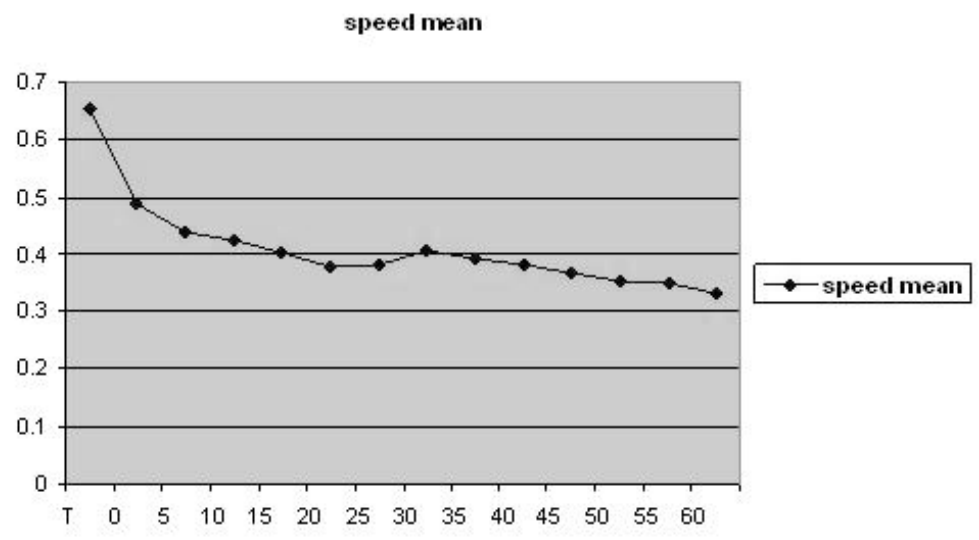

Figure 3.2 Relation between red (green) time and mean of speed

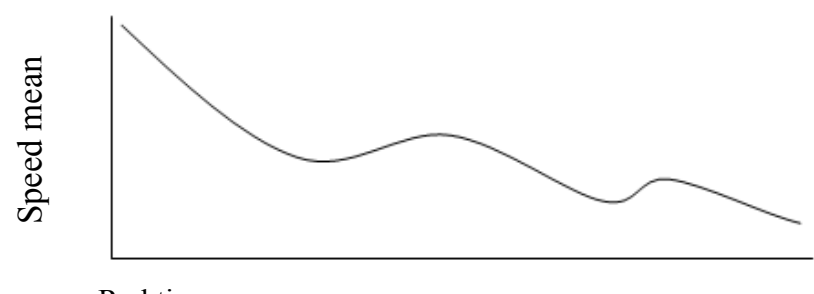

Red time

Figure 3.3 Anticipated curve 